\title{
Recent Developments in Simulations of Internal Flows in High Pressure Swirl Injectors
}

\author{
J. Cousin', W. M. Ren ${ }^{2}$ and S. Nally² \\ 1 Coria/UMR 6614 CNRS, Université et INSA de Rouen, Campus du Madrillet, 78801 Saint-Etienne du Rouvray - France \\ 2 Siemens Automotive, 615, Bland Boulevard, Newport News, VA 23602 - United States \\ e-mail: cousin@coria.fr
}

\begin{abstract}
Résumé - Progrès récents dans la simulation des écoulements dans les injecteurs haute pression à swirl - L'injecteur est un des éléments clés dans la conception des moteurs essence à injection directe. La modélisation de l'écoulement dans l'injecteur guide la conception de la géométrie interne. Dans une première partie, les aspects de cette modélisation seront soulevés, à savoir, l'écoulement transitoire en début et fin d'injection, ainsi que la prédiction des caractéristiques du carburant à la sortie de l'injecteur qui va conditionner les caractéristiques du spray. Dans une deuxième partie, nous évoquerons les théories d'instabilités à l'origine de la formation des sprays, ainsi que l'approche employée pour prédire les caractéristiques des gouttes générées.

Mots-clés : injecteurs, swirl, modélisation, spray.
\end{abstract}

\begin{abstract}
Recent Developments in Simulations of Internal Flows in High Pressure Swirl Injectors - As far as design of direct injection spark ignited engine is concerned, the fuel injector is a key component for success. Internal flow simulation is the best adapted tool for the design of the internal geometry of the injector. First, simulations of the internal flow will be discussed, especially transient stages of an injection event as well as the prediction of the fuel characteristics at the nozzle exit. Second, a coupling between internal flow calculations and instability model in order to predict initial spray characteristics will be suggested.

Keywords: Injector, Swirl, Modeling, Spray.
\end{abstract}

\section{INTRODUCTION}

Direct injection stratified charged (DISC) engine is actually recognized to be the best adapted in order to reduce both $\mathrm{No}_{x}$ emissions and fuel consumption in maintaining good engine performance. A correct achievement of this concept can be reached in combining lean combustion, improvement of thermodynamical output with increased compression ratio and reduced pumping loss.

In order to obtain a stratified charge, and therefore both globally lean mixture and locally reach mixture, late injection strategy is used at partial loads. With these conditions, time for the spray to evaporate is much shorter than in port fuel injection (PFI) concept. It is the reason why the spray quality and then the injector appears as a key factor in DISC engines.

High pressure swirl injectors are recognized to be one of the best adapted for the direct injection spark ignited engine because they generally provide a fine and widely dispersed fuel spray with moderate injection pressure.

Pressure swirl injectors that are extensively used in many industrial applications consist in inlet ports, a swirl chamber and a discharge orifice. During the injection process, pressurized liquid is forced to flow through tangential inlets into the swirl chamber, rotates in this chamber, and then emerges from the discharge orifice in the form of a thin conical sheet due to the creation of an air core inside the injector.

Many studies on pressure swirl injectors can be found in the literature [1]. Unfortunately, most of these studies are experimental and operating conditions are far from the present application. More precisely, for automotive applications, high values of injection pressure are necessary and short injection may be required and therefore unsteady flows have to be considered also. In this paper, a computational analysis of transient flows in high pressure swirl injectors is presented. 
Here, we are mostly interested in the temporal evolution of the emerging fuel that influences strongly the spray quality.

The next step consists in a linear analysis of the instabilities that are responsible for the breakup of the conical sheet. This analysis is briefly presented here and practical results are discussed as well.

\section{TRANSIENT FLOWS}

\subsection{Computational Model}

The flow that takes place in the atomizer is assumed to be fully turbulent and therefore the simulation is performed using the standard k-epsilon model. Figure 1 shows the classical computational domain and the grid when the needle that controls the amount of fuel to be discharged is at its upper position. Here only a quarter of injector is considered due to the symmetry of the injector.

Pressure boundary conditions are applied at the inlet and outlet planes. The inlet total pressure equals the fuel rail pressure (fixed at 70 bar for the simulation) and the relative outlet pressure equals zero. Cyclic boundary conditions are fixed at the two periodic planes. The Fire commercial code is used in this study where the movement of the needle is taken into account and the flow can be simulated during a complete injection event. In order to be as close as possible to the operation of a real injector, needle lift is deduced from experiments. Figure 2 illustrates the case of a $2 \mathrm{~ms}$ pulse width. Here it has to be kept in mind that the fully closed position can not be simulated and therefore injection is assumed to start and end with a small lift of $10 \mu$.

Dynamic calculations allow the prediction of the velocity field inside the injector as a function of time. Values of the instantaneous mass flow rate are directly deduced from these calculations and the procedure to predict the cone angle and the sheet thickness will be presented in detail.

\subsection{Understanding of the Flow}

In steady state operations, swirl atomizers are characterized by the cone angle $\theta$ and the discharge coefficient $C d$ defined by:

$$
C d=\frac{q_{m}}{\pi r_{0}^{2} \sqrt{2 \rho P i}}
$$

where $r_{0}$ and $P i$ are the orifice radius and the pressure differential respectively.

In Figure 3, classical evolutions of $C d$ and $\theta$ as a function of the injection pressure are reported. This figure is very helpful in understanding this particular flow and is directly linked to the characteristics of the fluid emerging from the nozzle as described by Lefebvre [1] (Fig. 3).

For very low injection pressure, the progression from the gutation regime (a) to the onion regime (c) passing through the jet regime (b) can be observed. These three regimes are characterized by liquid systems having a relative low velocity which results in a coarse atomization.

Between (a) and (b), $C d$ decreases because the injection pressure is not dissipated in friction only, but creates a "real" flow. At (c), $C d$ decreases slightly, this corresponds to the appearance of an air core around the axis of the chamber resulting from a high swirling motion. When $P i$ continues to increase, $C d$ and $\theta$ reach an asymptotic value and become independent of the injection pressure: the injector is said to work in its stable zone. This zone corresponds to the most effective mode of atomization because a large cone angle and a fine spray are supplied. This result was experimentally

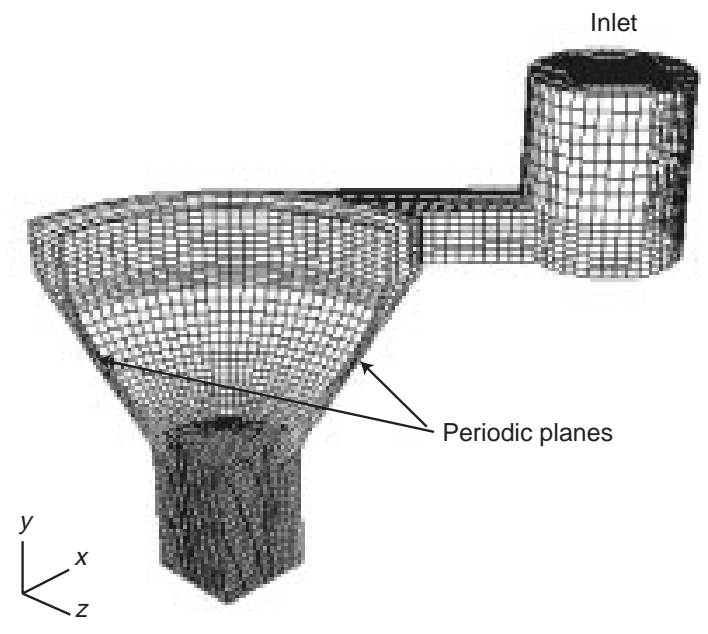

Figure 1

Computational domain and mesh.

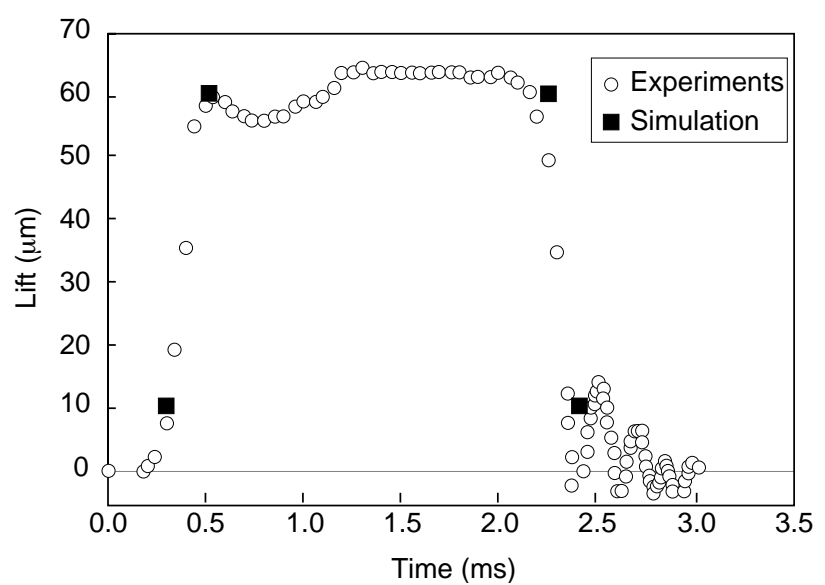

Figure 2

Simulation of the needle lift with time ( $T i=2 \mathrm{~ms})$. 


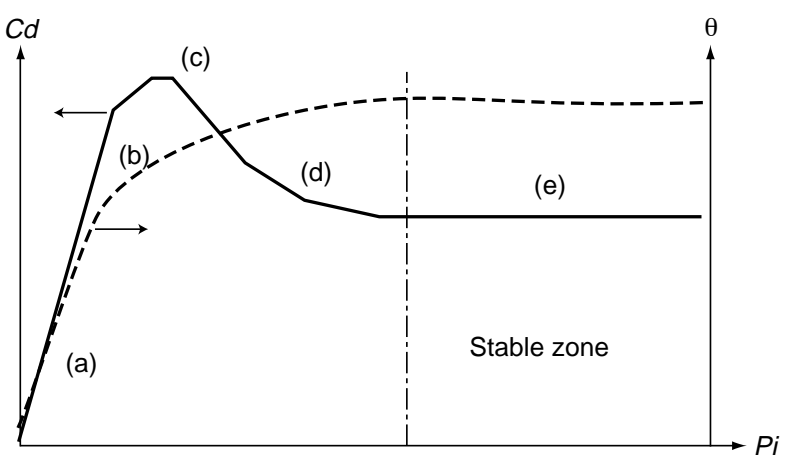

Figure 3

Evolution of $C d$ and $\theta$ with injection pressure.

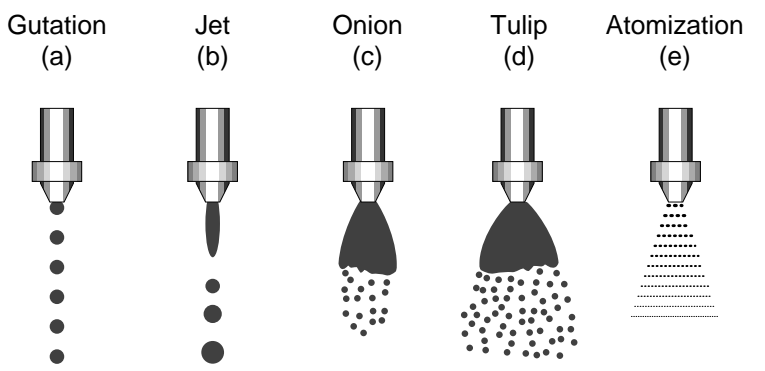

Figure 4

Behavior of the discharged fluid with $P i$.

observed by Doumas et al. [2] and Dombrowski et al. [3] who observed that $C d$ and $\theta$ depend only on the fluid properties and the injector design only. Typically, a good swirl injector is characterized by a small $C d$ (lower than .3) and a large cone angle in the stable zone. Moreover due to consideration of energetics, it is preferred to obtain the stable zone for small values of injection pressure.

In transient operating conditions, the behavior of the flow is similar to the description of Figures 2 and 3. At the start of injection, pressure below the sealing point equals zero. Then, injection pressure increases and the five regimes described previously are generally observed (Fig. 4). The quality of the spray resulting from the first four regimes is known to be undesirable compared to the stable zone.

\subsection{Results}

Figure 5 illustrates the typical evolution of the relative mass flow rate (mass flow rate/mass flow rate in steady state operation) with time. The curve is similar to what was described about evolution of discharge coefficient with injection pressure. At the start of injection, a high increase of relative mass flow rate $(\mathrm{qm})$ is observed. This peak corresponds to the discharge of a fuel jet at the orifice indicating a poorly atomized spray. Moreover, the stable zone is reached quickly and typically before the fully open position of the needle. This indicates that, as far as spray quality at the start of injection is concerned, movement of the needle is not a dominant parameter.

Although the air core is not taken into account in this study, an estimation is made possible with the help of the axial velocity at the orifice. As found in a previous study [4], a back flow is observed close to the axis of the injector and the air core is presumed to be located at the zero value of the axial velocity. Actually an experimental study is developed in order to validate the computed sheet thickness.

Figure 6 shows the temporal evolution of the sheet thickness at the orifice. At the start of injection, sheet thickness equals orifice radius indicating that a liquid jet is discharged at the injector tip. A sharp decrease of the sheet thickness is observed and finally its asymptotic value is reached quickly.

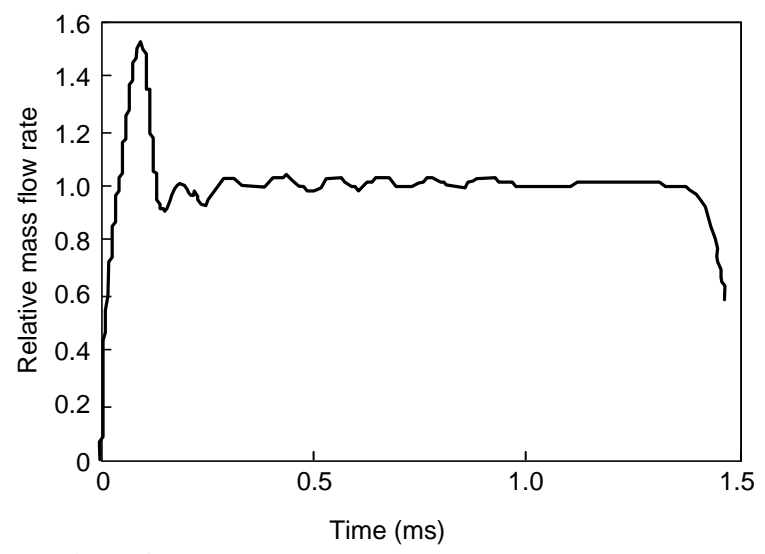

Figure 5

Temporal evolution of the mass flow rate.

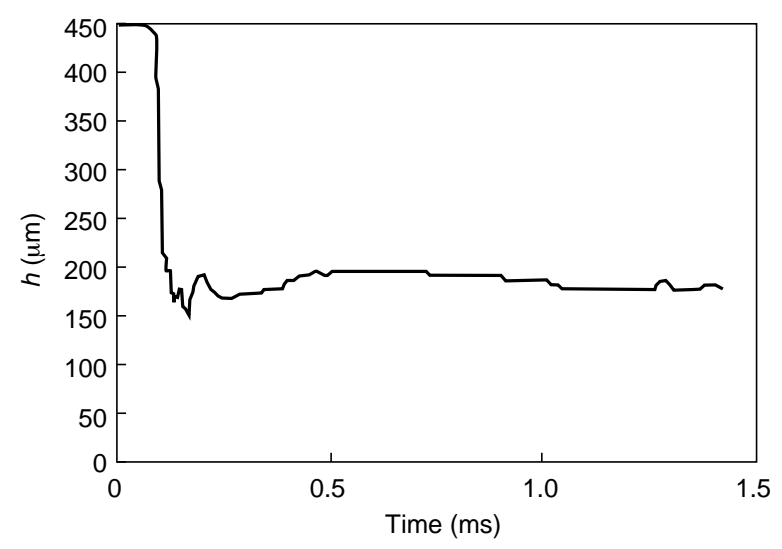

Figure 6

Temporal evolution of the sheet thickness. 
Mean axial and azimuthal velocities can be deduced when mass flow rate and sheet thickness are calculated. Mean axial velocity $W$ is deduced from a mass conservation equation and azimuthal velocity $V$ is deduced from the conservation of the angular momentum with respect to the chamber axis (see Cousin et al. [5] for details). Figure 7 shows the temporal evolution of the two mean velocities for the tested injector. It can be noticed that azimuthal velocity increases quicker than axial velocity indicating that the swirling flow is obtained quickly. At the end of injection, the opposite phenomenon is observed that is to say, when the needle fills up the orifice, $W$ decreases quicker than $V$. Then a slight increase of the cone angle is observed indicating that the spray quality remains correct. These results have been validated by measuring temporal evolution of the cone angle as shown in Figure 8.

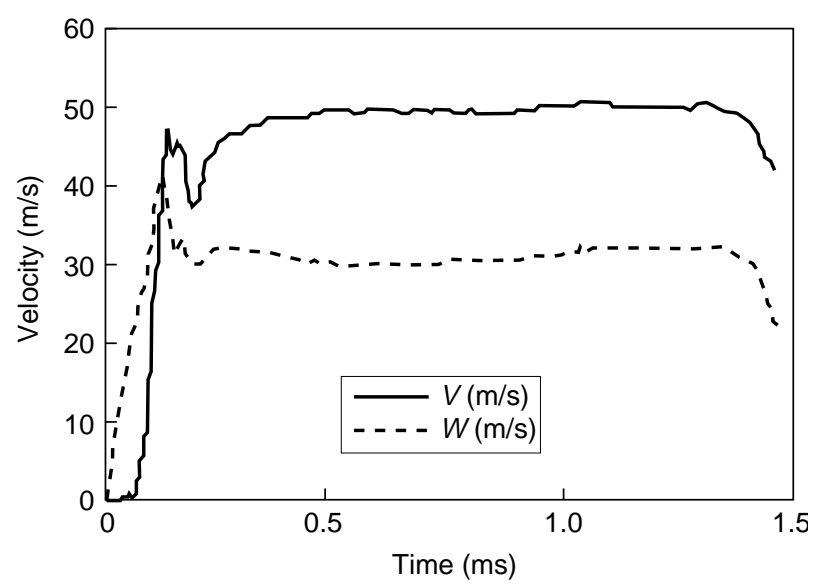

Figure 7

Temporal evolution of the mean exit velocities.

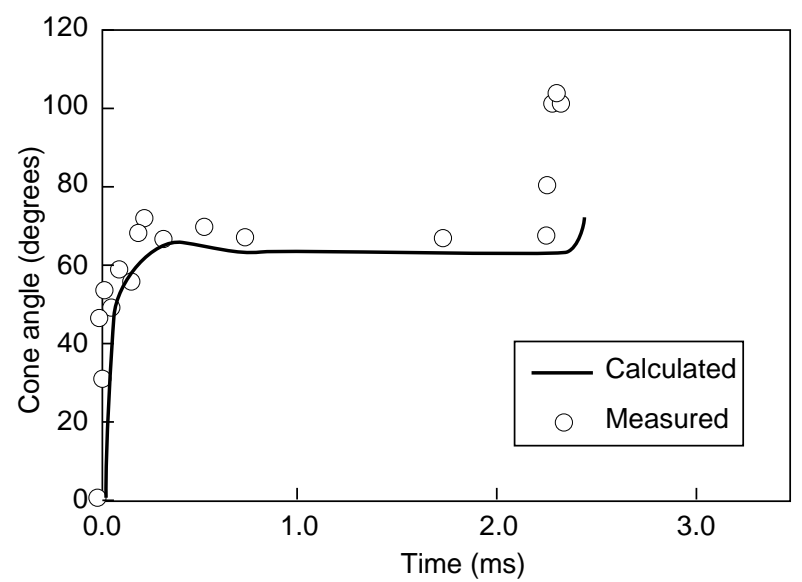

Figure 8

Evolution of calculated and measured cone angles.

\section{THE LINEAR THEORY}

\subsection{Introduction}

Up to now, linear theory is the most useful tool for the prediction of the characteristics of the unstable perturbation responsible for the breakup of liquid systems. By solving the linearized equations of the fluid mechanics on the interfaces separating the liquid and the gas, it is possible to characterize all the perturbations that may be present on the interface. Due to the calculation hypothesis, one can calculate the growth rate of each perturbation being defined by its wavelength. Finally due to the exponential growth of perturbation with time, perturbation that has the maximum growth rate and called dominant perturbation will be assumed to be the only responsible for the breakup.

\subsection{The Liquid Jet}

As mentioned in Section 1, during the first stage of an injection event, a liquid jet is discharged through the orifice. Then the linear theory is set on cylindrical jet having an axial velocity component deduced from previous internal flow simulations. Resolution of this application leads to the value of the dominant perturbation $k_{\max }$. By using single mass balance and by assuming that the jet is breaking each wavelength, a theoretical drop diameter $d_{G}$ may be calculated:

$$
d_{G}=\left(\frac{2 \pi r_{0}^{2}}{k_{\max }}\right)^{1 / 3}
$$

where $r_{0}$ is the orifice radius.

\subsection{The Conical Sheet}

Here, to reproduce the axisymmetry of the conical sheet, the linear theory is set for an annular liquid sheet with two components of velocity along the axis and the azimuth, as was done in a previous investigation [7]. Both the liquid and the gas are assumed to be incompressible and non viscous. Because of the two components of velocity, the perturbation, characterized by one wave number, may develop in both the axial and the azimuthal direction. The dominant wave that is assumed to be responsible for the disintegration of the conical sheet is our main interest here.

To apply this calculation to liquid systems discharged by pressure swirl atomizers, it is assumed that a conical sheet is comparable to a series of cylindrical sheets with increasing internal radius and decreasing thickness. The dominant wave is found to be the superposition of one axial perturbation of wave number $k_{\max }$ with an azimuthal perturbation of wave number $n_{\max / a}$ where a is the internal radius of the disintegrating liquid system. 
Then by using a disintegration scheme similar to the one suggested by Dombrowski and Hooper [8] for the case of a flat liquid sheet, a theoretical drop diameter can be calculated. Based on observations, this disintegration scheme suggests that the liquid sheet is breaking at each halfwavelength to form ligaments then behave as jets and disintegrate following a Rayleigh mechanism. By making simple mass balance, the theoretical drop diameter may be related to the dominant wave characteristics as well as to the internal radius $a$ and thickness $h$ of the disintegrating sheet at the breakup location. It yields :

$$
d_{G}=3.78 \sqrt{\frac{a h}{\sqrt{k_{\text {max }}^{2}+n_{\text {max }}^{2}}}}
$$

\subsection{Results}

Figure 9 gives the temporal evolution of $d_{G}$ during an injection event (pulse width $=2 \mathrm{~ms}$ ). One can observe the sharp decrease of $d_{G}$ at the start of injection due first to increasing axial velocity of the fuel jet and second to the change of liquid system geometry (from a jet to a conical sheet).

Comparisons with experimental data are not straightforward because $d_{G}$ is representative of the initial spray, that is to say just below the breakup plane. At this location, droplet size measurements are quite tricky due to the axial variations of the breakup plane. However for the same operating conditions, the Sauter mean drop diameter was found equal to $36 \mu, 1.5 \mathrm{~ms}$ after the start of injection. It has to be noticed that measurements performed with a PDPA system were located $50 \mathrm{~mm}$ downstream the injector tip and breakup location is estimated to be at $15 \mathrm{~mm}$ downstream.

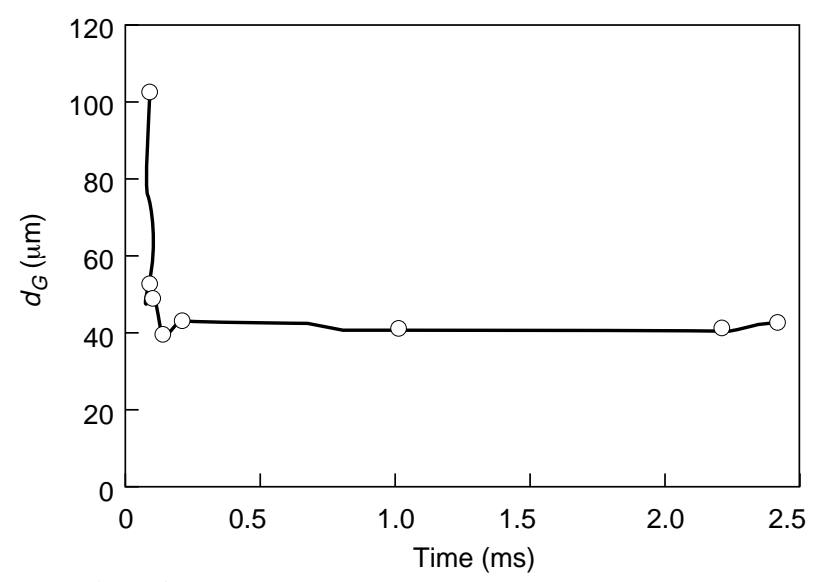

Figure 9

Evolution of the theoretical drop diameter.

\section{CONCLUSION}

The major conclusions of this work are listed below:

- Single phase flow simulation seems to give good results in terms of mass flow rate and cone angle.

Even if the air core is not taken into account, an approach is suggested to calculate the sheet thickness at the orifice. This parameter is fundamental here because it affects strongly the initial spray characteristics.

Actually, measurements of this thickness are performed and comparisons between calculations and measurements will be presented soon.

- Transient flow simulations have shown that the swirling flow is obtained very quickly and typically before the fully open position of the needle. This indicates that the time delay for the needle to reach its upper position is not a critical point for the start of an injection event.

- These results have allowed to reduce the mass of non swirling fuel at the start of an injection event, and therefore increasing the temporal spray quality. This has been made possible by modifying the internal geometry of the injectors.

- This paper also shows a method to couple internal flow simulations and instability models. This approach gives encouraging results and could be used as initial conditions for spray preparation modeling.

\section{REFERENCES}

1 Lefebvre, A.H. (1989) Atomization and Sprays, Hemisphere Publishing Corporation.

2 Doumas, M. and Laster, R. (1953) Liquid-Film Properties for Centrifugal Spray Nozzles. Chem. Eng. Prog., 518-526.

3 Dombrowski, N. and Hasson, D. (1969), AICHE Journ., 15, 4, 604 .

4 Ren, W.M., Shen, J. and Nally, J.F. (1997) Geometrical Effects on Flow Characteristics of Gasoline High Pressure Direct Injector. SAE Technical Paper No. 97FL-95.

5 Cousin, J., Ren, W.M., Nally, S. (1998) Transient Flows in High Pressure Swirl Injectors. SAE Technical Paper No. 980499.

6 Weber, C. (1931) Zum Zerfall eines Flussigkeitstrahles. Ztsehr Angew Math und Mech.

7 Dumouchel, C., Ledoux, M. (1991) Atomisation of Flat and Annular Sheets. Practical Use of Linear Theories. ICLASS'91 Paper No. 12.

8 Dombrowski, N., Hooper, P.C. (1962) The Effect of Ambiant Density on Drop Formation in Sprays. Chem Eng Scie, 13, 291-305. 